\title{
The future of cardiac transplantation
}

\author{
Kevin Koomalsingh, Jon A. Kobashigawa \\ Cedars-Sinai Heart Institute, Los Angeles, California, CA, USA \\ Correspondence to: Jon A. Kobashigawa, MD. Cedars-Sinai Heart Institute, 127 S. San Vicente Blvd, Los Angeles, California, CA 90048, USA. \\ Email: Kobashigawaj@cshs.org.
}

\begin{abstract}
The first human-to-human heart transplant was performed 50 years ago in 1967. Heart transplantation has now entered an era of tremendous growth and innovation. The future of heart transplantation is bright with the advent of newer immunosuppressive medications and strategies that may even result in tolerance. Much of this progress in heart transplant medicine is predicated on a better understanding of acute and chronic rejection pathways through basic science studies. The future will also include personalized medicine where genomics and molecular science will dictate customized treatment for optimal outcomes. The introduction of mechanical circulatory support (MCS) devices has changed the landscape for patients with severe heart failure to stabilize the most ill patient and make them better candidates for heart transplant. As ex vivo preservation takes hold, we may witness an expansion of the donor pool through the use of donation after cardiac death (DCD) donors. In addition, further geographical donor heart sharing through ex vivo preservation may further decrease waitlist mortality by enabling longer distance donor hearts to be allocated for the sickest waitlist patient. It is no doubt an exciting time to be involved in the field of heart transplantation. In this perspective, we will summarize the present state of heart transplantation and discuss various innovations that are being pursued.
\end{abstract}

Keywords: Cardiac transplantation; immunosuppression; rejection; mechanical circulatory support (MCS)

Submitted Aug 08, 2017. Accepted for publication Nov 25, 2017.

doi: $10.21037 /$ acs.2017.12.02

View this article at: http://dx.doi.org/10.21037/acs.2017.12.02

\section{Introduction}

The field of heart transplantation has made undeniable progress since the first human-to-human heart transplant was performed 50 years ago in 1967. It has now entered an era of tremendous growth and innovation. Over 67,000 heart transplants have been performed in the United States. This ranks third amongst solid organ transplantation. Since 1990, the heart transplant volume has averaged between 2,000-2,500 cases/year. Interestingly, over the past 3 years there has been a steady uptrend (Figure 1). In 2016, 3,190 heart transplants were performed. This is an exciting statistic and perhaps reflects increased donor utilization as the number of donors have remained stable. Survival after transplant has steadily improved with recent 1-, 5- and 10-year rates at approximately 90\%, 80\% and $65 \%$, respectively (1). In addition to the technological and pharmaceutical achievements, proper patient selection, superb medical management, meticulous operative care, vigilant surveillance and responsive long-term followup can all be credited for the continuing success in heart transplantation. Further advances in immunosuppression, immune tolerance and personalized medicine hold the key for future gains. In this perspective, we will summarize the present state of heart transplantation and discuss various innovations that are being pursued.

\section{Immunosuppression}

Much of the success of transplantation is due to the achievements in immunosuppression. Perhaps the biggest contributor was the introduction of calcineurin inhibitors (CNIs) in the 1980's. Since then, the presentday cocktail of a CNI (cyclosporine or tacrolimus), a purine inhibitor (azathioprine or mycophenolate) and a corticosteroid have been the cornerstone of heart transplant 


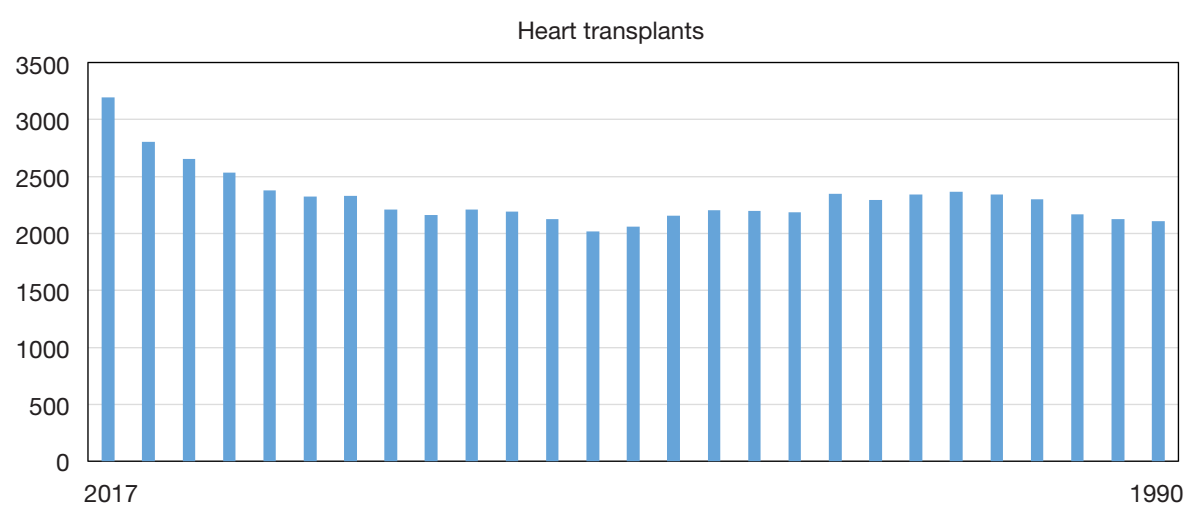

Figure 1 Heart transplant volumes in US. Notice the trend in recent years. UNOS data, 2017.

immunosuppression, but challenges remain. Debilitating side effects (nephrotoxicity), variable pharmacokinetics, malignancy and infection risks are valid long-term concerns. Attempts have been made to minimize the immunosuppression regimen, including steroid weaning, CNI monotherapy (2) and CNI-free immunosuppression (3), but has yet to become a standard of care.

The mammalian target of rapamycin (mTOR) plays an important role in cell growth and proliferation, especially in vascular smooth muscle cells and lymphocytes. Inhibitors of mTOR have demonstrated efficacy for rejection, allograft vasculopathy and even post-transplant malignancy (4-7). They have also been trialed in CNI free immunosuppression protocols (3). However, the augmentation of CNI nephrotoxicity and infectious complications with sirolimus and everolimus remain high and limit its use in routine immunosuppression. Another target is the CD28/B7 co-stimulatory pathway. Inhibitors of CD28/B 7 can potentially halt T-cell activation without the undesirable side effects of standard immunosuppression (8). Studies in animal models have been encouraging (9). The Food and Drug Administration (FDA) recently approved use of Belatacept in renal transplantation. Initial results are promising but infectious and malignancy complications appear concerning $(10,11)$. Advancements in pharmacogenomics can help elucidate the pharmacokinetics of maintenance immunosuppression. This will enable tailored dosing to maximize efficacy while minimizing side effects. It entails searching for polymorphisms and understanding specific gene expression profiles to customize immunosuppression for individual patients, also known as personalized medicine. For example, initial work with genomics in kidney transplantation has helped tailor tacrolimus dosing in African American recipients (12). This area of personalized medicine is being pursued in heart transplantation.

The field of immuno-biology continues to grow. It has resulted in an explosion of therapeutic antibodies. To date, several exist and are part of the pharmacologic armamentarium. These include: rituximab (anti-CD20), basiliximab (anti-IL2), alemtuzumab (anti-CD52), bortezomib (26S proteasome inhibitor), Belatacept (CD28/ B7 inhibitor) and eculizumab (anti-C5). Antibody therapy has facilitated management of challenging sensitized patients and even those suffering from cardiac allograft vasculopathy. Many antibodies are commonly utilized for induction therapy immediately after transplant, recalcitrant rejection and desensitization therapy.

Tolerance is the holy grail of organ transplantation. It involves the development of safe and effective regimens of manipulating the recipient immune system into accepting the transplanted organ in the absence of immunosuppressive therapy (13). Initial allograft tolerance studies with kidney transplants using donor bone marrow and non-myeloablative therapy showed stable peripheral blood mixed chimerism (the existence of two unrelated cell lines) in highly mismatched kidney transplants (14), which has resulted in these patients being successfully weaned off immunosuppression entirely. A similar strategy is also being investigated in heart transplantation, the results of which are being anxiously awaited for. Regulatory $\mathrm{T}$ cells have emerged with intense interest to avoid rejection and promote tolerance. They facilitate immune suppression to foreign antigens and therefore, can provide a solution to minimize or eliminate immunosuppressive therapy. If regulatory $\mathrm{T}$ cells can be coded to protect the graft from host immune responses, immunologic tolerance can be achieved. Initial animal studies have been encouraging (15) 
and human clinical trials are just underway (16). Studies have recently uncovered a regulatory role of $\mathrm{B}$ cells in transplant patients (17). Future studies will help elucidate this finding but it is also hoped to play a role in tolerance.

\section{Rejection}

Rejection is the "Achilles heel" of organ transplantation. It can manifest at any point after engraftment and the acuity can be varied. Generally, it is divided into cellular mediated rejection (CMR) and antibody mediated rejection (AMR). The tremendous progress in the field of transplant immunology has helped elucidate the cellular mediated pathways involved in organ transplantation and consequently, identify targets for induction and maintenance of immunosuppression (18).

AMR was initially at the forefront of transplantation in order to avoid the devastating consequences of hyperacute rejection. As the frequency of this complication dissipated, further research into the pathologic role of antibodies in transplantation laid relatively dormant. In fact, numerous endomyocardial biopsies demonstrating immunohistologic findings without clear evidence for CMR were either dismissed (if clinically asymptomatic) or labeled as biopsy-negative rejection (if there was evidence of graft dysfunction). In the past few years though, AMR has received intense scrutiny as its role in early (and late) graft loss is demonstrating increased clinical relevance. An International Society of Heart and Lung Transplantation (ISHLT) consensus conference was recently held to discuss this entity (19). Recommendations were established for diagnosis, surveillance and management.

New laboratory assays have enabled identification and quantification of antibodies against human leukocyte antigen (HLA) class I and class II antigens and even nonHLA antigens (20). The exact clinical significance of these antibodies is still being elucidated but their role in early and late graft loss is gaining significant traction. The recent advancement of linking donor-specific antibody (DSA) to complement fixation (C1q) is a significant leap forward for the field (21). Specific antibodies can now be evaluated for potential pathologic activity. This will have significant ramifications on virtual cross-matching, posttransplant surveillance protocols and even pre-emptive rejection treatment. As a result of these recent findings, our center is presently investigating the effectiveness of an anti-complement antibody in highly sensitized patients after heart transplantation (ClinicalTrials.gov identifier

\section{NCT02013037).}

Diagnosis of graft rejection requires a combination of pathologic, clinical and functional data. Endomyocardial biopsies will either occur at set intervals in asymptomatic patients, or urgently in symptomatic patients. Tissue samples are analyzed for histological and immunological findings. Clinically, patients are evaluated for signs and symptoms of heart failure while an objective graft assessment is done with echocardiography. Present management strategies for rejection include: altering maintenance dosing, pulsed steroids, induction agents, plasmapheresis, IVIG and hemodynamic support. As new pathways emerge, therapies will be tailored accordingly. The endomyocardial biopsy has been the cornerstone modality for identifying posttransplant rejection. However, the major limitations are the invasiveness of the procedure and subjectivity with pathological review. The CARGO II study uncovered a $28 \%$ concordance rate amongst panel pathologists reading $\geq 2 \mathrm{R}$ rejection (22). This has fueled efforts to discover a more reliable gold standard to detect rejection. One example, the AlloMap test (CareDX) is a biochemical serum assay assessing gene expression profiling to detect early cell-mediated rejection in post-transplant patients (23). Unfortunately, several limitations exist, such as a low positive predictive value, the inability to use the test within the first 2 months after transplant and the inability to detect AMR. However, it has demonstrated some utility and has solidified a position in the ISHLT guidelines. A very interesting study by Loupy et al., published this year, demonstrated a strong correlation between degree of graft injury and tissue based gene expression profiling for specific inflammatory cell mediators (24). Although there were some design limitations within the study, it represents an important step towards identifying a new gold standard for rejection diagnosis. Rather than a subjective pathology read on the heart biopsy, the Loupy study identifies molecular mechanisms for rejection in the biopsy sample which appears to be more reproducible and reliable. Studies in this area are ongoing. Additional areas of interest are donor derived cell-free DNA, microRNA and messenger RNA. They each strive to detect donor molecules that can infer an injury state or up-regulation of the immune system towards rejection.

\section{Cardiac allograft vasculopathy (CAV)}

CAV is a significant cause of late post-transplant mortality. Recent ISHLT registry data quotes rates of approximately $50 \%$ at 10 years (1). These rates have remained relatively 
unchanged over time and signals the need for focused attention to further improve long term outcomes. CAV is generally a diffuse coronary process characterized by concentric intimal thickening. It is thought to represent a form of chronic rejection. The pathogenesis is multifactorial and involves both immune and non-immune mechanisms. Cardiovascular risk factors such as diabetes, hypertension, hypercholesterolemia, hyperlipidemia and smoking are likely contributing culprits. Chronic inflammation and donor specific antibodies have a known association $(25,26)$ and are perhaps the larger instigators. The innate immune response is also suspected of mediating graft injury (27). Some have even theorized that ischemiareperfusion injury at the time of donor procurement alters gene expression, resulting in a rejection-susceptible protein milieu $(28,29)$. The use of mTOR inhibitors, statins and vitamin $\mathrm{C}$ and $\mathrm{E}$ have been demonstrated to slow the progression of $\mathrm{CAV}$, but to date, there is no therapy to completely prevent or reverse this significant complication. Future research can help unravel the science behind CAV and identify mechanisms to improve late survival. For now, angiographic monitoring, and earlier medical and interventional management may help temporize long-term graft loss.

\section{Mechanical circulatory support (MCS)}

In the past, patients awaiting heart transplantation with worsening heart failure had few options and had high mortality. Currently, MCS placement as a bridge to transplant (BTT) in these patients have made them more stable and hence better candidates for heart transplant. With a relatively fixed donor pool and a growing wait list, MCS is proving to be a valuable short and long-term solution for advanced heart failure. The durable device mortality rate has remained stable even as the implantation rates increase. However, complications such as bleeding, infections, strokes and pump thrombosis result in multiple readmissions and continue to plague this therapy. Single-center studies have reported readmission rates of $1.5-2.5$ per patient-year, with the majority occurring during the first 6 months after implantation $(30,31)$. The associated costs from these events are proving to be a significant financial burden on the health care industry. Most of the published figures however, are linked to the second-generation continuous flow (CF) left ventricular assist device (LVAD) such as the HeartMate II device. The third-generation devices (HeartMate III and HeartWare) are slowly approaching mainstream.
There is great optimism that the present technology will demonstrate lower complication rates (ENDURANCE, LATERAL and MOMENTUM trials).

As more durable devices are implanted, the BTT population will grow and wait list time will increase. Could this be inherently detrimental? This question was addressed by Colvin and colleagues in 2016. They simulated varying IA times for LVAD patients and looked at wait list mortality, transplant volume and post-transplant mortality. Surprisingly, allotting more IA time to LVAD patients did not significantly affect wait list mortality or post-transplant mortality (32). Stable LVAD patients have demonstrated remarkable outcomes with 2-year survival rates up to $70 \%$. Thus, these patients are less likely to benefit from a higher urgency status. However, subgroups such as extracorporeal membrane oxygenation (ECMO) patients or patients with an LVAD complication would be better suited for more urgent listing. These higher risk groups have become the focus for the new heart allocation system.

The new heart allocation system strives to provide the sickest patients with an organ in the shortest possible time. The goal is to reduce wait list and post-transplant mortality in the highest risk patients. It stratifies the MCS population into specific groupings based on urgency. This remedies the "subject to interpretation" dilemma with the existing system, which hopefully, will alleviate some of the bureaucratic processes. Lastly, it promotes geographic organ sharing. The changes have been fraught with concerns and criticisms. The impact will be seen shortly.

There has also been significant growth in the temporary mechanical support world as well. This includes the intraaortic balloon pump (IABP) (Maquet Wayne, NJ, USA), veno-arterial extracorporeal membrane oxygenation (VA-ECMO), Impella (Abiomed Danvers, MA, USA), TandemHeart (TandemLife Pittsburg, PA, USA) and CentriMag. There are increasing reports of the IABPs being inserted into the axillary artery for BTT (33). VAECMO continues to serve as a rescue therapy for critical cardiogenic shock and cardiac arrest. All-comer survival is $~ 50 \%$ (34). Ongoing clinical research with ECMO is helping to sketch out best practice patterns. For example, Cheng and colleagues found a double bridge time to durable device from VA-ECMO of less than 5 days to confer a significant mortality benefit (35). Furthermore, ECMO as BTT will be a class I priority in the new heart allocation scheme, thus expanding its future role. The Impella offers options for a variety of support ranging from left sided assist, to right sided assist to full left sided 
support via the Impella 2.5, Impella CP, Impella RP and Impella 5.0. The Impella 5.0 is gaining significant traction and proving to be an effective temporary support device. It provides excellent hemodynamic support, unloads the left ventricle and augments end organ perfusion. It also facilitates extubation and ambulation. Clinical data is still lagging but steadily emerging. Bansal et al. recently reported on their positive experience with 24 patients, where the Impella 5.0 was successfully used as a bridge to decision (36). The TandemHeart can provide either right or left sided support. It is most commonly used as an right ventricular assist device (RVAD) with the Protek cannula via the right internal jugular vein. The pump is limited to about $4.5 \mathrm{~L}$ flow but this is usually sufficient for right sided support. This too facilitates extubation and ambulation. Like the Impella, data is scarce, but reports have demonstrated significant hemodynamic improvements (37). These modalities have all proven to be versatile options for decompensated Interagency Registry for Mechanically Assisted Circulatory Support (INTERMACS) patients with end organ dysfunction. It will continue to enable innovative bridging pathways such as: bridge-to-transplant, bridge-todurable device, bridge-to-recovery, bridge-to-decision or even bridge-to-withdrawal. Future studies will help parse out specific patient-device-timing combinations to optimize outcomes. There has been a recent scientific statement on cardiogenic shock from the American Heart Association reviewing contemporary medical, surgical, mechanical circulatory support treatment approaches, and future research initiatives (38).

Optimism is growing for myocardial recovery. To date, LVAD as a bridge to recovery is an exceedingly rare event. It remains an after-thought with the focus being transplantation or destination therapy. However, WeverPinzon et al., using the INTERMACS database, showed that by designating patients upfront as possible bridge to recovery, the success of LVAD explantation can be as high as $25 \%$ (39). Similarly, in a recent study of 58 male patients with advanced heart disease, Jakovljevic et al. demonstrated that a significant percentage of LVAD explanted patients achieved a recovered functional capacity within the range of normal controls (40). Identifying appropriate patients and augmenting therapy with stem cells or tissue engineering can be ground-breaking.

\section{Ex vivo preservation}

Out of body organ preservation is not a new concept, but the application for the heart only recently completed the clinical trial period (PROCEED II). OCS Heart (TransMedics, Andover, MA, USA) is the first and only clinical ex vivo heart perfusion platform. It shortens cold ischemic time, permits ongoing metabolic and functional assessment and improves geographic organ sharing. In PROCEED II, 130 patients were randomized to OCS or standard cold storage. It was a non-inferiority study looking at 30-day patient and graft survival rates. The results were $94 \%$ vs. $97 \%$ with $\mathrm{P}=0.45$. The secondary endpoint (cardiac-related adverse events) was $13 \%$ vs. $14 \%$, also insignificant (41). Thus, OCS Heart demonstrated noninferiority to the present-day gold standard cold storage. This represents a huge leap for donor utilization and offers a promising future for heart procurement.

One of the visionary goals of heart transplantation has recently been achieved in the UK and Australia: donation after cardiac death (DCD) donors. Dhital and colleagues published their feasibility series of three DCD hearts in 2015. They used a technique of direct procurement and perfusion with the TransMedics OCS system (42). The following year, Messer and colleagues also demonstrated success with DCD hearts. They implanted nine DCD hearts after normothermic regional perfusion. The hearts were reperfused in situ. Metabolic and functional assessment were completed. The hearts were then arrested, explanted and transferred to the TransMedics OCS system. Finally, the hearts were re-evaluated using the same metrics and if deemed acceptable, were implanted. The authors reported a $100 \%$ survival rate without any detectable episodes of rejection (43). These landmark reports offer great optimism for the direction of donor utilization.

\section{Xenotransplantation}

Xenotransplantation offers a solution to the problem of donor shortages. Pig hearts are similar in size and anatomy to human hearts so are a favorable option. In addition to immunological risks from numerous antigens expressed by native porcine hearts, there is the risk of infection by porcine endogenous retroviruses (PERVs) which must be overcome for xenotransplantation to be feasible in clinical practice. PERVs are capable of being produced by porcine myocardial cells under stress which may infect human cells. A recent study by Yang et al. demonstrated successful inactivation of over 60 copies of PERV genes in the pig genome using the clustered regularly interspaced short palindromic repeats-Cas 9 (CRISPR-Cas 9) system (44). 
This recent development has resulted in a resurged interest in xenotransplantation, though further challenges must be overcome before clinical implementation. Survival in pig to non-human primate heart xenotransplant models is capped at 57 days. Histological analysis of explanted pig hearts shows that approximately $40-60 \%$ failed because of primary graft dysfunction rather than rejection (45).

\section{Limitations}

The benefit of various immunosuppressive regimens in clinical trials is limited by surrogate endpoints that are used to assert success. As there are relatively small number of heart transplant surgeries performed annually, there is inadequate power to demonstrate mortality benefit in any clinical trial. Therefore, biopsy-proven rejection has been used as the main surrogate endpoint in most trials. A major limitation in this endpoint is that concordance of pathology reads of rejection is only $67 \%$ due to various pathology artefacts that mimic rejection. Future endpoints for immunosuppression clinical trials might include other clinical outcomes (measure of cardiac dysfunction) and biomarkers (e.g., intragraft mRNA transcripts) that will predict good long-term outcomes.

A limitation for CAV therapy is that most clinical trials on CAV are predicated on first year intravascular ultrasound (IVUS) results. First year IVUS has not been validated through long-term follow up in this patient population. We await ongoing prospective trials to confirm that first year IVUS truly is an early marker for long-term outcomes.

A significant limitation to the use of mechanical circulatory support devices are its potential serious complications. These complications lead to frequent rehospitalizations, limiting the effectiveness of this therapy. At this time, the use of mechanical support devices will not replace heart transplantation for patients who are transplant candidates.

In the newer technologies involved with ex vivo perfusion and xenotransplantation there remains a great deal more to study which reduces enthusiasm. Although the CRISPRCas 9 system has revived interest in xenotransplantation, there remain significant obstacles. Successful gene therapy depends on a comprehensive understanding of underlying mechanisms of graft loss, which are still not fully understood in the pig to non-human primate setting.

\section{Conclusions}

In conclusion, the future of heart transplantation is bright with the advent of newer immunosuppressive medications and strategies that may result in tolerance (without need for immunosuppression). Much of this progress in heart transplant medicine is predicated on a better understanding of acute and chronic rejection pathways through basic science studies. The future will also include personalized medicine where genomics and molecular science will dictate customized treatment for optimal outcomes. The introduction of MCS devices has changed the landscape for patients with severe heart failure to stabilize the most ill patient and make them better candidates for heart transplant. This has resulted in lower waitlist mortality and improved post-transplant outcome for these patients. As ex vivo preservation takes hold, we may witness an expansion of the donor pool through the use of DCD donors. In addition, further geographical donor heart sharing through ex vivo preservation may further decrease waitlist mortality by enabling longer distance donor hearts to be allocated for the sickest waitlist patient. It is no doubt an exciting time to be involved with the field of heart transplantation. The frontier is being challenged on all sides-recipients, donors, immunosuppression, mechanical support, tolerance and genomics. The future will provide new explanations but likely reveal new challenges. Nevertheless, it is eagerly awaited.

\section{Acknowledgements}

None.

\section{Footnote}

Conflicts of Interest: JA Kobashigawa receives honoraria and research grants from Novartis AG, CareDx, Inc., and Transmedics Inc.; K Koomalsingh has no conflicts of interest to declare.

\section{References}

1. Lund LH, Edwards LB, Dipchand AI, et al. The Registry of the International Society for Heart and Lung Transplantation: thirty-third adult heart transplantation report-2016; focus theme: primary diagnostic tndications for transplant. J Heart Lung Transplant 2016;35:1158-69.

2. Baran DA, Zucker MJ, Arroyo LH, et al. A Prospective, Randomized Trial of Single-Drug Versus Dual-Drug Immunosuppression in Heart Transplantation. Circ Heart Fail 2011;4:129-37. 
3. Kushwaha SS, Khalpey Z, Frantz RP, et al. Sirolimus in cardiac transplantation: use as a primary immunosuppressant in calcineurin inhibitor-induced nephrotoxicity. J Heart Lung Transplant 2005;24:2129-36.

4. Keogh A, Richardson M, Ruygrok P, et al. Sirolimus in de novo heart transplant recipients reduces acute rejection and prevents coronary artery disease at 2 years. Circulation 2004;110:2694-700.

5. Clerkin KJ, Farr MA, Restaino SW, et al. Donor-specific anti-HLA antibodies with antibody-mediated rejection and long-term outcomes following heart transplantation. J Heart Lung Transplant 2017;36:540-5.

6. Majewski M, Korecka M, Joergensen J, et al. Immunosuppressive TOR kinase inhibitor everolimus (RAD) suppresses growth of cells derived from posttransplant lymphoproliferative disorder at allograftprotecting doses. Transplantation 2003;75:1710-7.

7. Kauffman HM, Cherikh WS, Cheng Y, et al. Maintenance immunosuppression with target-of-rapamycin inhibitors is associated with a reduced incidence of de novo malignancies. Transplantation 2005;80:883-9.

8. Kokko KE, Newell KA, Pearson TC, et al. Enhanced immunosuppression induced by targeted mutation of cytotoxic T lymphocyte antigen 4-immunoglobulin. Curr Opin Organ Transplant 2005;10:265-9.

9. Larsen CP, Pearson TC, Adams AB, et al. Rational development of LEA29Y (belatacept), a high-affinity variant of CTLA4-Ig with potent immunosuppressive properties. Am J Transplant 2005;5:443-53.

10. Vincenti F, Larsen C, Durrbach A, et al. Costimulation blockade with belatacept in renal transplantation. N Engl J Med 2005;353:770-81.

11. Grinyó J, Charpentier B, Pestana JM, et al. An integrated safety profile analysis of belatacept in kidney transplant recipients. Transplantation 2010;90:1521-7.

12. Jacobson PA, Oetting WS, Brearley AM, et al. Novel polymorphisms associated with tacrolimus trough concentrations: results from a multicenter kidney transplant consortium. Transplantation 2011;91:300.

13. Agarwal A, Ally W, Brayman K. The future direction and unmet needs of transplant immunosuppression. Expert Rev Clin Pharmacol 2016;9:873-6.

14. Leventhal J, Abecassis M, Miller J, et al. Chimerism and Tolerance Without GVHD or Engraftment Syndrome in HLA-Mismatched Combined Kidney and Hematopoietic Stem Cell Transplantation. Sci Transl Med 2012;4:124ra28.

15. Wood KJ, Sakaguchi S. Regulatory T cells in transplantation tolerance. Nat Rev Immunol 2003;3:199.

16. van der Net JB, Bushell A, Wood KJ, et al. Regulatory T cells: first steps of clinical application in solid organ transplantation. Transpl Int 2016;29:3-11.

17. Newell KA, Asare A, Kirk AD, et al. Identification of a B cell signature associated with renal transplant tolerance in humans. J Clin Invest 2010;120:1836-47.

18. Halloran PF. Immunosuppressive drugs for kidney transplantation. N Engl J Med 2004;351:2715-29.

19. Colvin MM, Cook JL, Chang P, et al. Antibodymediated rejection in cardiac transplantation: emerging knowledge in diagnosis and management. Circulation 2015;131:1608-39.

20. Tinckam KJ, Chandraker A. Mechanisms and role of HLA and non-HLA alloantibodies. Clin J Am Soc Nephrol 2006;1:404-14.

21. Chen G, Sequeira F, Tyan DB. Novel C1q assay reveals a clinically relevant subset of human leukocyte antigen antibodies independent of immunoglobulin G strength on single antigen beads. Hum Immunol 2011;72:849-58.

22. Crespo-Leiro MG, Zuckermann A, Bara C, et al. Concordance Among Pathologists in the Second Cardiac Allograft Rejection Gene Expression Observational Study (CARGO II). Transplantation 2012;94:1172-7.

23. Pham MX, Teuteberg JJ, Kfoury AG, et al. GeneExpression Profiling for Rejection Surveillance after Cardiac Transplantation. N Engl J Med 2010;362:1890-900.

24. Loupy A, Duong Van Huyen JP, Hidalgo L, et al. Gene Expression Profiling for the Identification and Classification of Antibody-Mediated Heart Rejection. Circulation 2017;135:917-35.

25. Reed EF, Demetris AJ, Hammond E, et al. Acute Antibody-mediated Rejection of Cardiac Transplants. J Heart Lung Transplant 2006;25:153-9.

26. Kobashigawa J, Crespo-Leiro MG, Ensminger SM, et al. Report from a consensus conference on antibodymediated rejection in heart transplantation. J Heart Lung Transplant 2011;30:252-69.

27. Kobashigawa JA. The Future of Heart Transplantation. Am J Transplant 2012;12:2875-91.

28. Li XC. The significance of non-T cell pathways in graft rejection--implications for transplant tolerance. Transplantation 2010;90:1043.

29. Kim IK, Bedi DS, Denecke C, et al. Impact of innate and adaptive immunity on rejection and tolerance. Transplantation 2008;86:889-94.

30. Hasin T, Marmor Y, Kremers W, et al. Readmissions after implantation of axial flow left ventricular assist device. J 
Am Coll Cardiol 2013;61:153-63.

31. Forest SJ, Bello R, Friedmann P, et al. Readmissions After Ventricular Assist Device: Etiologies, Patterns, and Days Out of Hospital. Ann Thorac Surg 2013;95:1276-81.

32. Colvin M, Miranda-Herrera D, Gustafson SK, et al. Impact of increased time at the highest urgency category on heart transplant outcomes for candidates with ventricular assist devices. J Heart Lung Transplant 2016;35:326-34.

33. Russo MJ, Jeevanandam V, Stepney J, et al. Intra-aortic balloon pump inserted through the subclavian artery: A minimally invasive approach to mechanical support in the ambulatory end-stage heart failure patient. J Thorac Cardiovasc Surg 2012;144:951-5.

34. Nichol G, Karmy-Jones R, Salerno C, et al. Systematic review of percutaneous cardiopulmonary bypass for cardiac arrest or cardiogenic shock states. Resuscitation 2006;70:381-94.

35. Cheng R, Ramzy D, Azarbal B, et al. Device Strategies for Patients in INTERMACS Profiles 1 and 2 Cardiogenic Shock: Double Bridge With Extracorporeal Membrane Oxygenation and Initial Implant of More Durable Devices. Artif Organs 2017;41:224-32.

36. Bansal A, Bhama JK, Patel R, et al. Using the Minimally Invasive Impella 5.0 via the Right Subclavian Artery Cutdown for Acute on Chronic Decompensated Heart Failure as a Bridge to Decision. Ochsner J 2016;16:210-6.

37. Kar B, Gregoric ID, Basra SS, et al. The percutaneous ventricular assist device in severe refractory cardiogenic

Cite this article as: Koomalsingh K, Kobashigawa JA. The future of cardiac transplantation. Ann Cardiothorac Surg 2018;7(1):135-142. doi: 10.21037/acs.2017.12.02 shock. J Am Coll Cardiol 2011;57:688-96.

38. van Diepen S, Katz JN, Albert NM, et al. Contemporary Management of Cardiogenic Shock: A Scientific Statement From the American Heart Association. Circulation 2017;136:e232-68.

39. Wever-Pinzon O, Drakos SG, McKellar SH, et al. LVAD Explantation for Cardiac Recovery: Insights from the Interagency Registry for Mechanically Assisted Circulatory Support. J Heart Lung Transplant 2016;35:S11.

40. Jakovljevic DG, Yacoub MH, Schueler S, et al. Left Ventricular Assist Device as a Bridge to Recovery for Patients With Advanced Heart Failure. J Am Coll Cardiol 2017;69:1924-33.

41. Ardehali A, Esmailian F, Deng M, et al. Ex-vivo perfusion of donor hearts for human heart transplantation (PROCEED II): a prospective, open-label, multicentre, randomised noninferiority trial. Lancet 2015;385:2577-84.

42. Dhital KK, Iyer A, Connellan M, et al. Adult heart transplantation with distant procurement and ex-vivo preservation of donor hearts after circulatory death: a case series. Lancet 2015;385:2585-91.

43. Messer SJ, Axell RG, Colah S, et al. Functional assessment and transplantation of the donor heart after circulatory death. J Heart Lung Transplant 2016;35:1443-52.

44. Niu D, Wei HJ, Lin L, et al. Inactivation of porcine endogenous retrovirus in pigs using CRISPR-Cas9. Science 2017;357:1303-7.

45. Cowan PJ, Tector AJ. The resurgence of xenotransplantation. Am J Transplant 2017;17:2531-6. 